\title{
Association of the HSPG2 Gene with Neuroleptic-Induced Tardive Dyskinesia
}

\author{
Aoi Syu ${ }^{1,2}$, Hiroki Ishiguro',2, Toshiya Inada ${ }^{3}$, Yasue Horiuchi ${ }^{1,2}$, Syunsuke Tanaka', Maya Ishikawa', \\ Makoto Arai ${ }^{4}$, Masanari Itokawa ${ }^{4}$, Kazuhiro Niizato ${ }^{5}$, Shuji Iritani ${ }^{5}$, Norio Ozaki ${ }^{6}$, Makoto Takahashi ${ }^{7}$, \\ Akiyoshi Kakita $^{8}$, Hitoshi Takahashi ${ }^{8}$, Hiroyuki Nawa ${ }^{8}, K^{2}$ azuko Keino-Masu', Eri Arikawa-Hirasawa ${ }^{10}$ \\ and Tadao Arinami*,1,2
}

'Department of Medical Genetics and Department of Molecular Neurobiology, Graduate School of Comprehensive Human Sciences, University of Tsukuba, Tsukuba, Ibaraki, Japan; ${ }^{2}$ CREST, Japan Science and Technology Agency, Kawaguchi-shi, Saitama, Japan; ${ }^{3}$ Institute of

Neuropsychiatry, Seiwa Hospital, Tokyo, Japan; ${ }^{4}$ Department of Schizophrenia Research, Tokyo Institute of Psychiatry, Tokyo, Japan; ${ }^{5}$ Department of Psychiatry, Tokyo Metropolitan Matsuzawa Hospital, Tokyo, Japan; ${ }^{6}$ Department of Psychiatry, School of Medicine, Nagoya University, Nagoya, Aichi, Japan; ${ }^{7}$ Department of Psychiatry, Niigata University Graduate School of Medical and Dental Sciences, Niigata, Japan; ${ }^{8}$ Brain Research Institute, Niigata University, Niigata, Japan; ${ }^{9}$ Department of Molecular Neurobiology, Graduate School of Comprehensive Human Sciences, University of Tsukuba, Tsukuba, Ibaraki, Japan; ${ }^{10}$ Research Institute for Diseases of Old Age, Department of Neurology, Juntendo University School of Medicine, Tokyo, Japan

\begin{abstract}
Tardive dyskinesia (TD) is characterized by repetitive, involuntary, and purposeless movements that develop in patients treated with long-term dopaminergic antagonists, usually antipsychotics. By a genome-wide association screening of TD in 50 Japanese schizophrenia patients with treatment-resistant TD and 50 Japanese schizophrenia patients without TD (non-TD group) and subsequent confirmation in independent samples of 36 treatment-resistant TD and 136 non-TD subjects, we identified association of a single nucleotide polymorphism, rs2445142, (allelic $p=2 \times 10^{-5}$ ) in the HSPG2 (heparan sulfate proteoglycan 2, perlecan) gene with TD. The risk allele was significantly associated with higher expression of HSPG2 in postmortem human prefrontal brain $(p<0.0 \mathrm{I})$. Administration of daily injection of haloperidol (HDL) for 50 weeks significantly reduced Hspg2 expression in mouse brains $(p<0.00 \mathrm{I})$. Vacuous chewing movements (VCMs) induced by 7-week injection of haloperidol-reserpine were significantly infrequent in adult Hspg2 hetero-knockout mice compared with wild-type littermates $(p<0.00 \mathrm{I})$. Treatment by the acetylcholinesterase inhibitor, physostigmine, was significantly effective for reduction of VCMs in wild-type mice but not in Hspg2 hetero-knockout mice. These findings suggest that the HSPG2 gene is involved in neuroleptic-induced TD and higher expression of HSPG2, probably even after antipsychotic treatment, and may be associated with TD susceptibility.

Neuropsychopharmacology (20 I0) 35, I I55-I I64; doi: I0.1038/npp.2009.220; published online I3 January 2010
\end{abstract}

Keywords: acetylcholine; neurogenetics; schizophrenia/antipsychotics; pharmacogenetics/pharmacogenomics; tardive dyskinesia

\section{INTRODUCTION}

Antipsychotic-induced tardive dyskinesia (TD) is an involuntary movement disorder that develops in patients who are undergoing long-term treatment with antipsychotic medications. The clinical symptoms most commonly involve orobuccal, lingual, and facial muscles, especially in older individuals. The lingual involvement in the form of fine vermicular movements of the tongue while it is sitting

*Correspondence: Professor $\mathrm{T}$ Arinami, Department of Medical Genetics, Graduate School of Comprehensive Human Sciences, University of Tsukuba, I-I-I Tennoudai, Tsukuba, Ibaraki 305-8575, Japan, Tel: + 8I-29-853-3177, Fax: + 81-29-853-3333,

E-mail: tarinami@md.tsukuba.ac.jp

Received 20 May 2009; revised 6 November 2009; accepted 23 November 2009 at the base of the oral cavity is a common early feature (Sachdev, 2000). In more severe cases, the movements may involve trunk and limbs (Tarsy and Baldessarini, 2006). Such movements lower the quality of life (QOL) of patients (Gerlach, 2002). Therefore, predicting those patients who are vulnerable to TD remains a high priority for psychiatrists in selecting the best medication for a given individual. Introduction of second-generation atypical antipsychotics has reduced the occurrence of TD to approximately $1 \%$ annually compared with the $5 \%$ frequency with typical agents (de Leon, 2007; Remington, 2007). Owing to the lack of effective treatments for TD, however, therapeutic management of TD can be problematic for schizophrenia patients receiving antipsychotic medications, especially for those patients who develop severe treatment-resistant TD. Therefore, the strategies to prevent TD are often discussed 
in the context of safety and use of antipsychotic drugs (Inada et al, 2008).

The etiology of TD is complex and remains unclear. Age, gender, and ethnicity are all the suggested risk factors for TD. Smoking, drinking, and using street drugs may also increase the risk of TD (Menza et al, 1991). There is some evidence for a genetic component to TD (Muller et al, 2004) and molecular genetic studies of TD were conducted to identify genes related to TD (Malhotra et al, 2004).

The pathophysiology of TD is not completely understood. The causative role of antipsychotic and other dopamine antagonists resulted in the proposal of the dopamine supersensitivity hypothesis of TD (Klawans et al, 1980). However, as the hypothesis explains only some aspects of TD, many other pathophysiological models including changes in other neurotransmitter signaling systems that are affected by neuroleptics have been considered. They include gamma-aminobutyric acid (Gerlach and Casey, 1988), norepinephrine (Saito et al, 1986), serotonin (Haleem, 2006), and acetylcholine (Ach) (Tammenmaa et al, 2002).

The advent of single nucleotide polymorphism (SNP) chips for genome-wide association analysis has made screening of susceptibility genes for TD possible. We carried out a genome-wide association study of treatmentresistant TD in schizophrenia patients and reported that SNPs associated with TD were aggregated significantly in genes belonging to the gamma-aminobutyric acid receptor signaling pathway (Inada et al, 2008). In this study, we analyzed the HSPG2 gene, which includes SNPs that showed the most significant association with TD in our genomewide association study.

\section{MATERIAL AND METHODS}

\section{Ethical Considerations}

This study was initiated after approval by the ethics committee of each institution. Written informed consent was obtained from all patients after adequate explanation of the study.

\section{Human Subjects}

Human subjects in this study were 86 Japanese schizophrenia patients with TD and 186 Japanese schizophrenia patients without TD, who have been described elsewhere (Inada et al, 2008). Briefly, subjects were identified at psychiatric hospitals located around Tokyo and Nagoya areas of Japan. All patients satisfied the diagnostic criteria of DSM-IV (Association, 1994) for schizophrenia. All subjects and their parents were of Japanese descent. All subjects had been receiving antipsychotic therapy for at least 1 year and their TD status was monitored for at least 1 year. TD was assessed according to the Japanese version of the Abnormal Involuntary Movement Scale. TD was diagnosed according to the criteria proposed by Schooler and Kane (Schooler and Kane, 1982). Once TD was identified, the patients were followed up and received standard therapeutic regimens for TD to minimize TD symptoms. If TD persisted even after 1 year of therapy, patients were considered potential treatment-resistant TD patients. Treatment-resistant TD patients were defined as those patients with dyskinetic movements that persisted for more than 1 year and did not improve even after 1 year of appropriate treatment after guideline-recommended therapeutic regimens for TD. We hypothesized that treatment-resistant TD, a severe form of TD, was suitable for detection of genetic association with TD. Only treatmentresistant TD patients were included as those affected with TD in this study.

\section{Genotyping, Resequencing, and Statistics}

Association screening was performed using the Illumina Sentrix Human-1 Genotyping 109k BeadChip according to the manufacturer's instructions (Illumina, San Diego CA, USA). All DNA samples were subjected to rigorous quality control to check for fragmentation and amplification. Approximately $750 \mathrm{ng}$ of genomic DNA was used in each sample. Normalized bead intensity data obtained for each sample was entered into the Illumina BeadStudio 3.0 software, which converted fluorescence intensities into SNP genotypes. A GenCall Score of 0.85 was used as a minimum threshold for per-sample genotyping completeness. The mean call rate across all samples was $97 \%$. After removing SNPs with a low genotyping rate $(p<0.95$ : $n=3952)$, SNPs deviating from the Hardy-Weinberg equilibrium $(p<0.001: n=135)$, SNPs with low minor allele frequency (MAF < 0.05: $n=2762$ ), and SNPs located outside exons and introns, we screened for SNPs associated with TD using 40573 SNPs. SNPs located within $10 \mathrm{~kb}$ from the $5^{\prime}$ and $3^{\prime}$ ends of known genes were included. SNPs in the linkage disequilibrium (LD) of $r^{2}>0.8$ with other SNPs were excluded. The call rate was at least $99.4 \%$ for the 40573 SNPs. The concordance rate was evaluated by comparisons of genotypes in the 100 screening samples and this gave concordance of over $98 \%$ for each sample. Genotyping using TaqMan probes (Applied Biosystems, Foster City, CA, USA) was carried out twice for each SNP, and genotype concordance was $99.7 \%$. Genotyping completeness was $>0.99$. We treated these uncalled or discrepant genotypes as missing genotypes.

To screen for novel polymorphisms, we used direct sequencing with a Big Dye Terminator Cycle Sequencing kit and ABI PRISM 3100 Genetic Analyzer (Applied Biosystems, Foster City, CA, USA). All exons and the exon-intron junctions of the HSPG2 gene were amplified from the genomic DNAs of the 86 TD group patients. The sequences of primers for mutation screening are available on request.

For a more detailed analysis of the associations between SNPs in the HSPG2 gene and TD, the tag SNPs in the gene were selected using the Haploview program (http:// www.broad.mit.edu/mpg/haploview/) with the condition of an $r^{2}$ threshold of 0.8 and a minor allele frequency of 0.1 , and genotyped by the TaqMan method. Allelic discrimination was performed using the ABI PRISM 7900HT Sequence Detection System using SDS 2.0 software (Applied Biosystems, Foster City, CA, USA).

Allelic associations between SNPs and TD, and departure from the Hardy-Weinberg equilibrium were evaluated by $\chi^{2}$ test or Fisher's exact test. Bonferroni's correction for multiple comparisons was applied. 


\section{Human Postmortem Brains}

Brain specimens were from individuals of European (Australian) and Japanese descent. The Australian sample comprised 10 schizophrenic patients and 10 age- and gender-matched controls. The diagnosis of schizophrenia was made according to the Diagnostic and Statistical Manual of Mental Disorders (DSM)-IV criteria (American Psychiatric; Association (1994)) by a psychiatrist and a senior psychologist. Control subjects had no known history of psychiatric illness. Tissue blocks were cut from gray matter in an area of the prefrontal cortex referred to as Brodmann's area 9 (BA9). Japanese samples of BA9 gray matter from Japanese brain specimens consisted of six schizophrenic patients and 11 age- and gender-matched controls. In addition, postmortem brains of 37 deceased Japanese patients with schizophrenia were also analyzed. The Japanese subjects met the DSM-III-R criteria for schizophrenia. Details of the condition of the postmortem brains have been described elsewhere (Ishiguro et al, 2008; Koga et al, 2009).

\section{Analysis of Hspg2 Transcription in Human Brain Tissue}

Total RNA was extracted from human brain tissues with ISOGEN Reagent (Nippon Gene, Tokyo, Japan). The RNA quality was checked using a Nanodrop ND-1000 spectrophotometer (LMS, Tokyo, Japan) to have an OD 260/280 ratio of $1.8-2$ and an OD $260 / 230$ of 1.8 or greater. Expression of the HSPG2 genes was analyzed by the TaqMan real-time polymerase chain reaction system (Applied Biosystems, Foster City, CA). From RNA, cDNA was synthesized with Revertra Ace (Toyobo, Tokyo, Japan) and oligo dT primers. Expression of the HSPG2 gene was analyzed with an ABI PRISM 7900 HT Sequence Detection System (Applied Biosystems), with the TaqMan gene expression assays for HSPG2 (Hs01078535_m1), and normalized to the expression of Human GAPDH Control Reagents (Applied Biosystems).

Genotype effects on HSPG2 expression were analyzed in Australian subjects and replicated in Japanese subjects using analysis of variance followed by Tukey's post hoc tests by JMP software version 7.0.1 (SAS Institute, Cary, NC, USA) was used.

\section{Animals}

Animals were same-sex housed before behavior testing. The same animals were used for all behavior tests.

Four-week-old C57BL/6J male mice (weight: 20-25 g) treated with haloperidol (HDL) or vehicle-saline and 7-week-old male mice (wild type: 8; Hspg2 ${ }^{+/-}: 7$ ) with orofacial dyskinesia were housed under $10 \mathrm{~h}: 14 \mathrm{~h}$ light/dark conditions with normal food and water ad libitum, with mice housed separately in groups of 4 or 5 mice.

The generation of Hspg2 knockout mice and the phenotypes of the mice have been described elsewhere (Arikawa-Hirasawa et al, 1999). As Hspg2 null mice are embryonic lethal, timed matings between heterozygotes were carried out to generate homozygous and wild-type mice in this study.
All animal protocols were approved by the Animal Care and Use committee of University of Tsukuba.

\section{Drugs}

Reserpine (methyl reserpate 3,4,5-trimethoxycinnamic acid ester; Wako, Osaka, Japan) and HDL (Wako, Osaka, Japan) were diluted in glacial acetic acid and then diluted in distilled water. Physostigmine (Wako), a reversible cholinesterase inhibitor, was diluted in saline. All solutions were treated subcutaneously in volumes not exceeding $10 \mathrm{ml} / \mathrm{kg}$ body weight.

\section{HDL Treatment}

To examine the effects of antipsychotic treatments on gene expression, we made two groups: an acute treatment group: 4-week-old C57BL/6J male mice were treated with intraperitoneal injection (i.p.) of $1.0 \mathrm{mg} / \mathrm{kg} \mathrm{HDL}(n=10)$ or vehiclesaline $(n=10)$ once each day for 4 weeks; and a long-term treatment group: 4-week-old C57BL/6J male mice were treated with intraperitoneal injection of $1.0 \mathrm{mg} / \mathrm{kg} \mathrm{HDL}$ $(n=10)$ or vehicle-saline $(n=10)$ once each day for 50 weeks. Mice were killed $4 \mathrm{~h}$ after the last injection to obtain brain tissues.

\section{Induction of Vacuous Chewing Movements}

Mice were treated with i.p. of $2 \mathrm{mg} / \mathrm{kg} \mathrm{HDL}$ and $0.3 \mathrm{mg} / \mathrm{kg}$ reserpine every day for 7 weeks to induce the putative TD analogue vacuous chewing movements (VCMs) (Araujo et al, 2004; Burger et al, 2005; Naidu et al, 2003). Before injection and 4 hours after the injection on the 47 th day, locomotor activity test and rotarod test were carried out. On the 48th and 49th days, 1, 2, 3, 4, and $24 \mathrm{~h}$ after the last injection, the animals were observed for quantification of VCMs for 2 days. On the 50th day, to verify the effects of physostigmine on VCMs, mice were injected with $0.1 \mathrm{mg} / \mathrm{kg}$ physostigmine. At 1, 2, 3, 4, and $24 \mathrm{~h}$ after the injection of physostigmine, the animals were observed for quantification of VCMs. On the 51st and 52nd day, mice were treated with $2 \mathrm{mg} / \mathrm{kg} \mathrm{HDL}$ and $0.3 \mathrm{mg} / \mathrm{kg}$ reserpine and then observed for quantification of VCMs. On the 53rd day, mice were treated with vehicle-saline, and 1, 2, 3, 4, and $24 \mathrm{~h}$ after the injection, the animals were observed for quantification of VCMs.

\section{Analysis of Hspg2 Transcription in Brain Tissue of Mice}

The prefrontal cortex, midbrain, hippocampus, thalamus, and striatum were taken by dissection, and total RNA was extracted with an RNeasy kit (Qiagen, K.K., Tokyo, Japan). After cDNA synthesis from total RNA samples, the transcription level of cDNA samples was analyzed by a TaqMan Expression assay for Hspg2 (Mm00464581_m1; Applied Biosystems) and normalized to that of rodent Gapdh with Rodent Gapdh Control Reagents (Applied Biosystems). The average relative expression levels of five regions were compared with the saline groups by Student's $t$-test. 
Table I Allelic p-Values of SNPs for Association with TD in Screening and Replication Samples

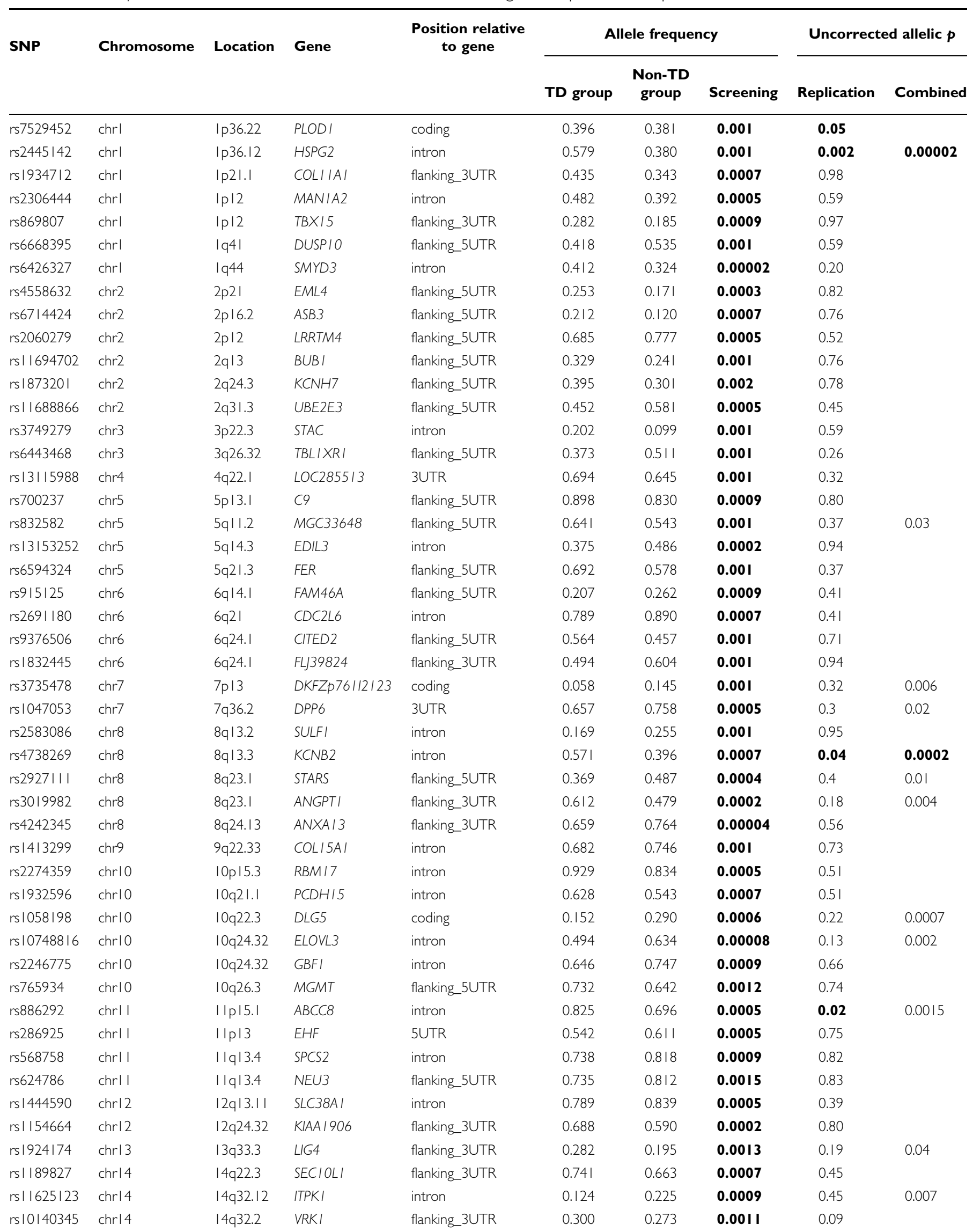


Table I Continued

\begin{tabular}{|c|c|c|c|c|c|c|c|c|c|}
\hline \multirow{2}{*}{ SNP } & \multirow{2}{*}{ Chromosome } & \multirow{2}{*}{ Location } & \multirow{2}{*}{ Gene } & \multirow{2}{*}{$\begin{array}{l}\text { Position relative } \\
\text { to gene }\end{array}$} & \multicolumn{3}{|c|}{ Allele frequency } & \multicolumn{2}{|c|}{ Uncorrected allelic $p$} \\
\hline & & & & & TD group & $\begin{array}{l}\text { Non-TD } \\
\text { group }\end{array}$ & Screening & Replication & Combined \\
\hline rs206105I & chrl5 & $15 q \mid 2$ & GABRG3 & intron & 0.206 & 0.350 & 0.0014 & 0.04 & 0.0006 \\
\hline rs376421I & chrl5 & $15 q \mid 3.1$ & APBA2 & flanking_3UTR & 0.726 & 0.815 & 0.0013 & 0.12 & 0.005 \\
\hline rs4630608 & chrl7 & $|7 p| 1.2$ & $F B \times W I O$ & intron & 0.250 & 0.274 & 0.0010 & 0.04 & \\
\hline rs2287352 & chrl7 & $17 q 12$ & ACACA & flanking_5UTR & 0.247 & 0.306 & 0.0014 & 0.85 & \\
\hline rs3744I 65 & chrl7 & 17q25.3 & FLJ I 384 | & 5UTR & 0.093 & 0.130 & 0.0010 & 0.15 & \\
\hline$r s 474122$ & $\operatorname{chrl} 8$ & $|8 p||.3|$ & DLGAPI & flanking_5UTR & 0.404 & 0.330 & 0.0002 & 0.38 & \\
\hline rs 12460403 & chrl9 & $19 p \mid 3.3$ & HMG2OB & flanking_3UTR & 0.285 & 0.194 & 0.0011 & 0.55 & \\
\hline rs2328500 & chr20 & $20 p \mid 1.23$ & C20orf26 & intron & 0.376 & 0.324 & 0.001 & 0.12 & \\
\hline rs7281019 & chr2I & $21 \mathrm{q} 22.11$ & TCPIOL & intron & 0.924 & 0.862 & 0.00008 & 0.57 & 0.04 \\
\hline rs2056965 & chr22 & $22 q \mid 2.3$ & LOC9/464 & flanking_5UTR & 0.422 & 0.348 & 0.0002 & 0.49 & \\
\hline
\end{tabular}

Abbreviations: SNP, single nucleotide polymorphism; TD, tardive dyskinesia.

$p$-Values with bold emphasis indicate $p<0.05$ in Ist $p$ and 2 nd $p$, and combined $p<I$ st $p$.

\section{Evaluation of VCMs}

Mice were placed individually in observation cages $\left(16 \times 17 \times 19 \mathrm{~cm}^{3}\right)$ without food. Hand-operated counters were used to quantify VCMs continuously for $5 \mathrm{~min}$. VCMs were referred to as single mouth openings in the vertical plane not directed toward physical material. If VCMs occurred during a period of grooming, they were not taken into account. Mirrors were placed under the floor and behind the back wall of the cage to permit observation of oral movements when the animal faced away from the observer. The observations were made by two observers who were blind to the animal's group assignment. The observation criteria were not subjective, because an excellent inter-observer agreement was found in a previous pilot experiment (Pearson's correlation $=0.98$ ). All behavioral experiments were conducted between 1000 and 1800hours.

\section{Locomotor Activity}

The locomotor activity test was conducted between 1200 and 1700 hours in a dimly lit testing room. Mice were habituated to the room for at least $30 \mathrm{~min}$ before testing. The locomotor activity test was videotaped with a Sony Digital Video Camera (Sony, Tokyo, Japan). The behavioral testing apparatus was a black Plexiglas rectangular box $(41 \mathrm{~cm}$ long $\times 22 \mathrm{~cm}$ wide $\times 20.5 \mathrm{~cm}$ tall $)$ and activity was recorded for $20 \mathrm{~min}$. The total distance traveled (locomotion) was scored.

\section{Rotarod Test}

The rotarod test was conducted between 1200 and 1700 hours in a dimly lit testing room. All mice were brought to the testing room in their home cages and were allowed to sit undisturbed in the testing room for at least $5 \mathrm{~min}$ before the start of behavioral testing. Motor performance was assessed by rotarod (Med Associates, St Albans, VT). A 1-min training session was given to each mouse on the rotarod (diameter $8 \mathrm{~cm}, 7 \mathrm{rpm}$ ) $5 \mathrm{~min}$ before the first measurement. Motor performance (time until the first fall) was registered during a 2 -min session.

\section{Statistical Analysis for Behavioral Data and Gene Expression in Animal Experiments}

Effects of genotype, drug treatment, and time were analyzed using analysis of variance) followed by Tukey's post hoc tests or using Student's $t$-test. Individual differences of the number of VCMs between before and after injection of physostigmine and saline were tested by nonparametric test for one sample test of mean $=0$.

\section{RESULTS}

\section{Association Study}

We screened for SNPs associated with TD using 40573 tag SNPs on the Sentrix ${ }^{\circledR}$ Human-1 Genotyping BeadChip (Illumina) to identify loci associated with susceptibility to TD in 50 TD and 50 non-TD subjects (Inada et al, 2008). The potential impact of population structure on this association study was evaluated by using the genome-wide $\chi^{2}$ inflation factor, $\lambda$, as a genomic control (Devlin and Roeder, 1999; Devlin et al, 2001). The estimated value of $\lambda$ was 1.04 , by which genome-wide association $p$-values were corrected. The lowest uncorrected allelic $p$-value for association with TD was $1 \times 10^{-5}$. Therefore, no SNP was 
significantly associated with TD after Bonferroni correction. An attempt was made to replicate the association of 63 SNPs, which were allelic $p$-values $<0.002$ and located within $10 \mathrm{~kb}$ from known genes with the TaqMan genotyping assay (Table 1). A potential association was found for four SNPs (allelic $p<0.05$ ) (Table 1). However, no significant association was found after correction for multiple testing of 63 SNPs in the replication sample only. Among these four SNPs, an association between GABRG3 SNP and TD has already been reported (Inada et al, 2008). The lowest allelic $p$-value for the association was found for $\mathrm{rs} 2445142$ $\left(p=2 \times 10^{-5}\right)$ when the initial genome-wide sample and replication sample were combined. The SNP is located in the HSPG2 gene.

Next, we tested associations of 24 tag SNPs including rs 2445142 in the HSPG2 gene with TD and found a nominal significant association for five SNPs in addition to rs2445142 (Table 2). Other than rs2445142, we found a significant association of rs 2124368 located in intron 43 of the HSPG2 gene with TD even after applying Bonferroni's correction for multiple testing (uncorrected allelic $p=0.0003$, corrected $p=0.007$ ). The SNP rs2124368 was not in LD with rs2445142, which was located in intron 1 $\left(D^{\prime}=0.13, r^{2}=0.01\right)$.

Subsequently, we genotyped the SNPs of rs 2501255 (intron 1), rs2501257 (intron 1), rs897474 (intron 3), rs2254357 (exon 6), rs2254358 (exon 6), and rs2497632 (intron 9), because of the expected complete LD between these SNPs and rs2445142 based on the HapMap database. We confirmed that they were in complete LD with rs2445142 $\left(r^{2}=1.00\right)$. These SNPs were located in introns 1-9 of the HSPG2 gene. Age, sex, and age of onset were not associated with these SNPs. Acute extrapyramidal symptoms were associated with rs 2445142 and the six SNPs in complete LD with rs 2445142 (allelic $p=0.00002$ ). Resequencing all exons of the HSPG2 gene in patients with TD did not reveal novel SNPs. Finally, we genotyped missense SNPs of rs3736360, rs2229493, s2291827, rs2228349, rs2229491, rs2229490, rs2229489, rs2229475, rs897471, rs2229481, and rs989994, which were listed in dbSNP and did not find significant associations of these SNPs with TD (data not shown). Thus, we tested a total of 103 SNPs, including 41 SNPs in the HSPG2 gene, in our total subjects of $86 \mathrm{TD}$ and 136 non-TD patients.

\section{Association Between Hspg2 Expression Levels in the Postmortem Prefrontal Cortex and Rs2445142}

The transcription level in the postmortem prefrontal cortex, as measured by TaqMan real-time polymerase chain reaction, was not significantly different by diagnosis, age, sex, postmortem intervals, or $\mathrm{pH}$ of brain samples. A significant genotype effect on HSPG2 gene expression levels was observed in 20 Australian subjects $(\mathrm{F}(2,17)=4.9$, $p=0.02)$ and replicated in 54 Japanese subjects $(\mathrm{F}(2,51)=3.5, p=0.04)$. The association was significant in the combined subjects $(\mathrm{F}(2,71)=7.6, p=0.001)$. Tukey's post hoc tests showed that HSPG2 expression levels were significantly higher in the subjects with the GG genotype than in those with the CC genotype (Figure 1). Unfortunately, information about TD in the brains we analyzed was not available.
Table 2 Allelic p-Values of Tag SNPs in the HSPG2 Gene for Association with TD

\begin{tabular}{|c|c|c|c|c|c|}
\hline & \multirow{2}{*}{ Location } & \multicolumn{4}{|c|}{ Allele frequency* } \\
\hline & & Allele & $\begin{array}{l}\text { TD } \\
\text { group }\end{array}$ & $\begin{array}{l}\text { Non-TD } \\
\text { group }\end{array}$ & Allelic $p$ \\
\hline rs3736360 & exon 96 (N433IS) & $A / G$ & 0.19 & 0.20 & $0.87 \mid 5$ \\
\hline rs3767|37 & intron 77 & $A / G$ & 0.23 & 0.19 & 0.2759 \\
\hline rs109|7053 & intron 7l & $A / G$ & 1.00 & 0.99 & 0.3308 \\
\hline rs7355045 & intron 64 & $G / A$ & 0.84 & 0.81 & 0.4235 \\
\hline rs229050I & intron 60 & C/A & 0.22 & 0.21 & 0.7134 \\
\hline rs I563370 & intron 52 & $A / G$ & 0.35 & 0.27 & 0.0687 \\
\hline rs2229475 & exon 47 (II967V) & $\mathrm{G} / \mathrm{A}$ & 0.01 & 0.01 & 0.9477 \\
\hline rs2305562 & intron 43 & $A / G$ & 0.61 & 0.49 & 0.0117 \\
\hline rs465499| & intron 42 & $\mathrm{G} / \mathrm{A}$ & 0.39 & 0.36 & 0.5605 \\
\hline rs2124368 & intron 42 & G/A & 0.77 & 0.60 & 0.0003 \\
\hline rs897472 & intron 36 & C/A & 0.09 & 0.05 & 0.1098 \\
\hline rs89747| & exon 36 (VI503A) & $A / G$ & 0.88 & 0.87 & 0.7005 \\
\hline rs2229478 & exon 8 (L248L) & $A / G$ & 0.53 & 0.42 & 0.0273 \\
\hline rs3767|4I & intron 6 & $\mathrm{G} / \mathrm{A}$ & 0.66 & 0.60 & 0.1811 \\
\hline rs2445I42 & intron I & $\mathrm{G} / \mathrm{C}$ & 0.58 & 0.38 & 0.00002 \\
\hline rs878949 & intron I & $A / G$ & 0.22 & 0.20 & 0.5867 \\
\hline rs I545593 & intron I & C/A & 0.41 & 0.30 & 0.0122 \\
\hline rs 1002480 & intron I & $\mathrm{G} / \mathrm{C}$ & 0.41 & 0.32 & 0.0368 \\
\hline rs6698486 & intron I & $\mathrm{G} / \mathrm{A}$ & 0.46 & 0.38 & 0.0754 \\
\hline rs 10799719 & intron I & G/A & 0.80 & 0.75 & 0.1789 \\
\hline rs9426785 & intron I & $A / G$ & 0.57 & 0.55 & 0.7389 \\
\hline rs4654773 & intron I & $A / G$ & 0.45 & 0.45 & 0.9165 \\
\hline rs| I587857 & intron I & G/A & 0.50 & 0.46 & 0.3465 \\
\hline rs4233280 & $5^{\prime}$ flanking & $A / G$ & 0.07 & 0.03 & 0.0588 \\
\hline
\end{tabular}

Abbreviations: SNP, single nucleotide polymorphism; TD, tardive dyskinesia

*The frequency of the first allele.
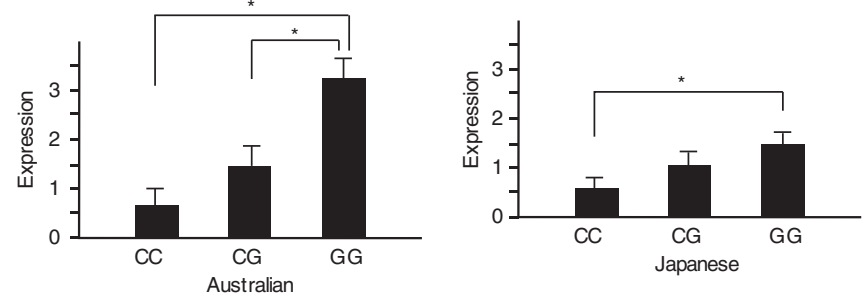

Figure I HSPG2 expression levels in the postmortem prefrontal region by genotype. The vertical scores show the average (SEM) of relative expression levels in each of the three genotype groups, compared with the mean gene expression in the total samples. *Indicates $p<0.05$ by Tukey's post hoc tests.

\section{Hspg2 Gene Expression in the Mouse Brains by HDL Treatment}

Hspg2 expression levels were evaluated in the mouse brain after treatment with the antipsychotic drug, HDL. The expression of Hspg2 levels did not alter after a 4-week treatment of HDL except for the striatum where Hspg2 was expressed significantly higher than after the saline 

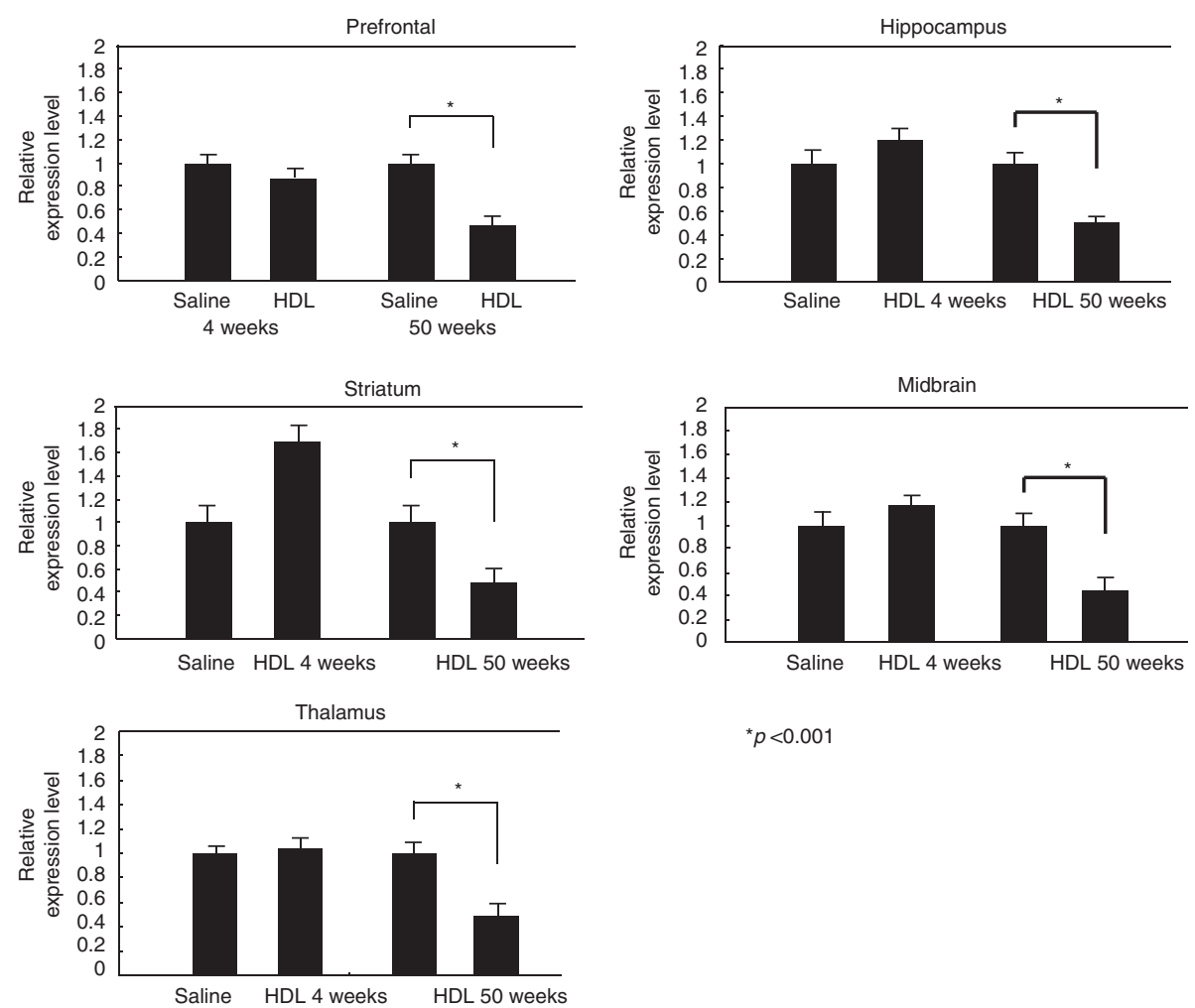

${ }^{*} p<0.001$

Figure 2 Effects of haloperidol (HDL) on HSPG2 gene expression in the mouse brains. Relative expression levels of Hspg2 from the prefrontal cortex, midbrain, hippocampus, thalamus, and striatum in the mouse brains after treatment with HDL for 4 weeks $(n=10)$ and HDL for 50 weeks $(n=10)$ were compared with the saline groups for 4 weeks $(n=10)$ and 10 weeks $(n=10)$ by Student's $t$-test.

treatment. Significantly lower expression of Hspg2 was observed in all brain regions after a 50-week treatment with HDL than after a 50-week treatment with saline (Figure 2) $(\mathrm{F}(1,18)=42.9, p<0.0001$ at the prefrontal cortex; $\mathrm{F}(1,18)=$ $20.1, p=0.0003$ at the hippocampus; $\mathrm{F}(1,18)=15.9, p=0.0009$ at the striatum; $\mathrm{F}(1,17=19.3, p=0.0004$ at the midbrain; $\mathrm{F}(1,18)=16.5, p=0.0007$ at the thalamus).

\section{Analysis of VCMs Induced by Haloperidol-Reserpine in Hspg2 Knockout Mice}

As we could not induce VCMs by administration of HDL only to mice, VCMs induced by long-term treatment with HDL and reserpine in female Hspg2 hetero-knockout mice and female wild-type gene litters were measured to evaluate the relationship between expression levels of Hspg2 and TD (Figure 3a). Hspg2-null knockout mice were embryonic lethal. The relative expression levels of Hspg2 in Hspg2 hetero-knockout mouse brains were almost half of that in the wild littermates (data not shown). Body weight, locomotor activities, and performance in the rotarod test before and after 48 days of administration of HDL and reserpine were not significantly different between Hspg2 hetero-knockout and wild litters (data not shown). There was a significant effect of genotype $(\mathrm{F}(1,545)=36.8$, $p<0.0001)$, post-treatment time $(\mathrm{F}(4,495)=6.15$, $p<0.0001)$, and treatment $(\mathrm{F}(3,543)=5.7, p=0.0008)$ for the number of VCMs for $5 \mathrm{~min}$. Post hoc analysis showed that the number of VCMs were significantly lower in hetero-knockout mice than in wild-type mice after the last injection of HDL and reserpine after 48 or 49 consecutive days of administration of HDL and reserpine, and subsequent injection of physostigmine on the 50th day, or saline on the 53rd day (Figure 3b). The response of VCMs to physostigmine was subsequently evaluated (Figure 3c). There was a significant effect of genotype $(\mathrm{F}(1,128)=36.9$, $\mathrm{p}<0.0001)$, but not post-treatment time $(\mathrm{F}(4,125)=1.03$, $\mathrm{p}=0.39$ ) for individual differences in the number of VCMs between pre-injection and post-treatment time. As for saline treatment, there was no significant effect of genotype $(\mathrm{F}(1,118)=0.13, p=0.72)$ and post-treatment time $(\mathrm{F}(4,115)=0.31, p=0.87)$. The numbers of VCMs were significantly reduced by injection of physostigmine compared with those before the injection at $24 \mathrm{~h}$ after HDL and reserpine injection in the wild-type mice but the differences in the numbers of VCMs before and after injection of physostigmine were not significant in hetero-knockout mice. The number of VCMs did not significantly alter after injection of saline in hetero-knockout mice and wild-type mice.

\section{DISCUSSION}

From a genome-wide association analysis, this study identified the role of HSPG2 in neuroleptic-induced TD. The association was not significant in the initial screening and second confirmation after correction for multiple testing. However, screening with the tag SNPs for HSPG2, 
Evaluation of locomotor and rota-rod activities

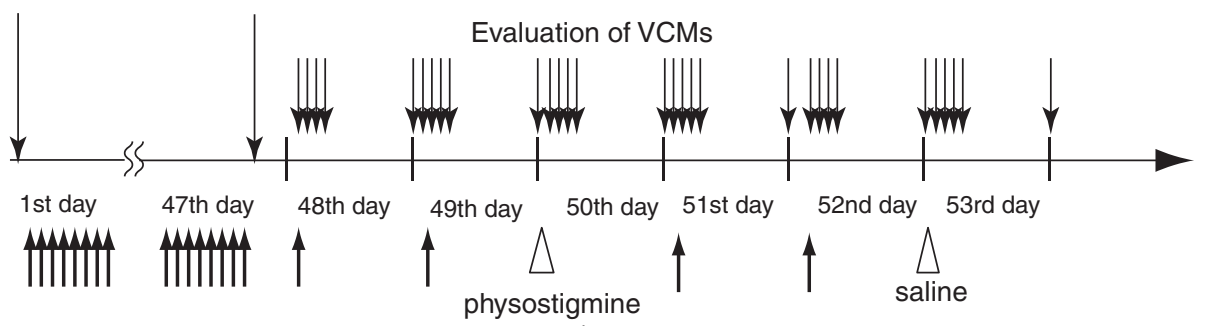

$0.1 \mathrm{mg} / \mathrm{kg}$

haloperidol $2 \mathrm{mg} / \mathrm{kg}$ + reserpine $0.3 \mathrm{mg} / \mathrm{kg}$

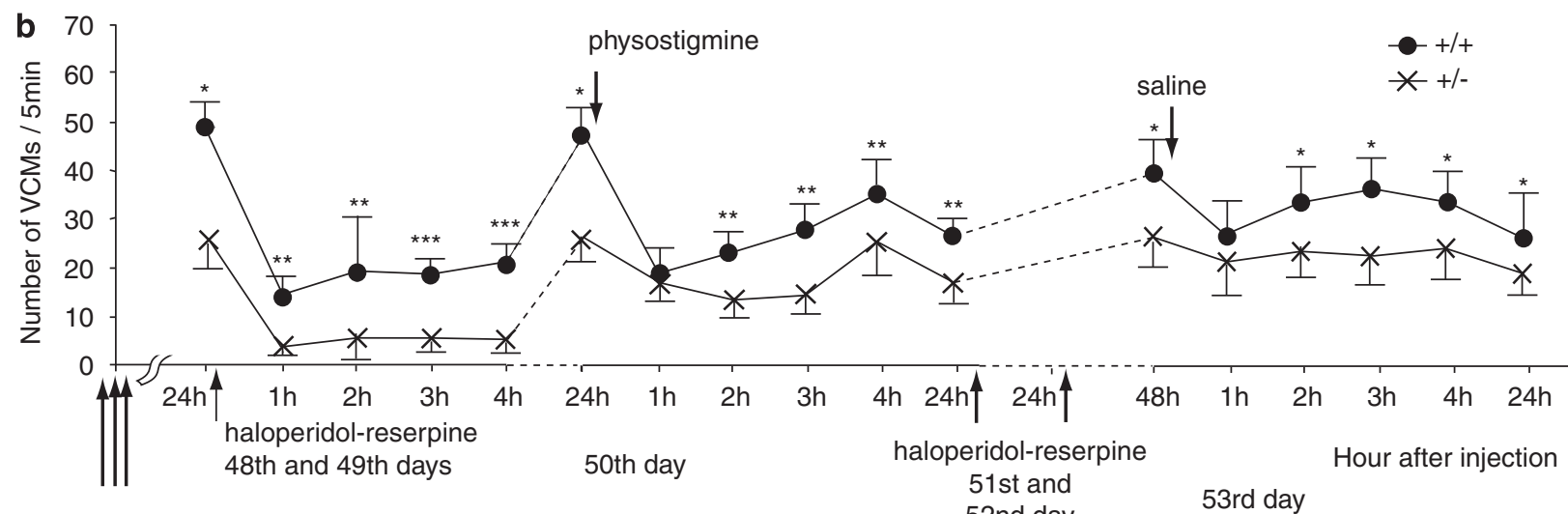

47 or 48 days consecutive injection of haloperidol-reserpine

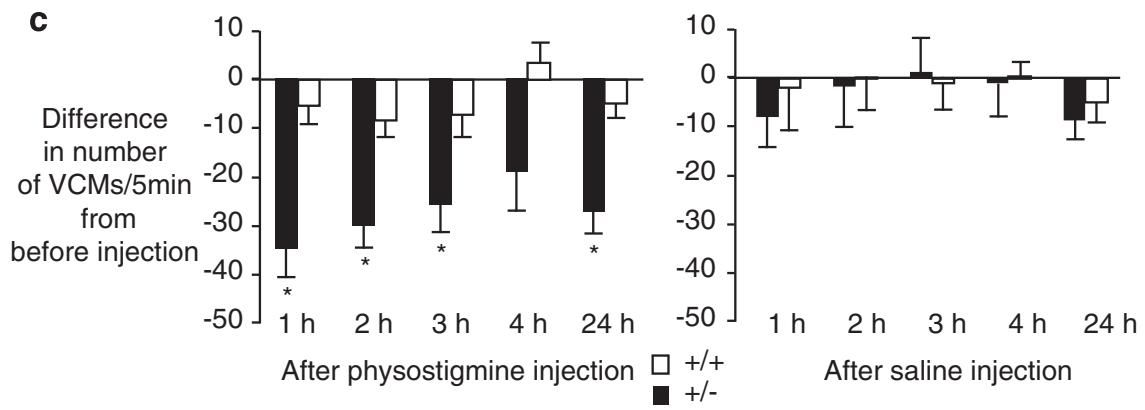

Figure 3 Analysis of vacuous chewing movements (VCMs) induced by haloperidol-reserpine in Hspg2 knockout mice. (a) Schematic presentation of schedule of injections and measurements. (b) The average number (SEM) of VCMs for 5 min after injection. The abscissa axis shows the time after injection of HDL and reserpine, physostigmine, or saline. Significant difference between Hspg2 hetero-knockout and wild-type mice is shown as * at $p<0.05$, ** at $p<0.01$, and $* * *$ at $p<0.001$. (c) Reduction of the number of VCMs after physostigmine or saline injection. Individual differences of the number of VCMs before injection (50th day for physostigmine or 53rd day for saline) to each time after injection grouped by the genotype are shown. Significant difference from mean $=0$ is shown as $*$ at $p<0.001$.

where the SNP (rs2445142) with the smallest $p$-value for association with TD in our genome-wide association study was located, identified one SNP (rs2124368) associated with TD even after correction for multiple testing. These two SNPs, which were found to be associated with TD, one identified by a genome-wide screening and another identified by screening with the tag SNPs, were not in LD. However, it is not obvious whether the finding for genetic association with TD of these SNPs in the HSPG2 gene can be interpreted as significant, because of two steps of genomewide association analyses before the step of screening of tag SNPs. Furthermore, the Human-1 BeadChip used in our initial screening is far from a complete genome coverage.
This may affect the credibility of the results. Confirmation of associations in other populations is necessary.

The SNP rs2445142 that showed the lowest association $p$-value in this study was associated with the expression levels of HSPG2 in the human postmortem prefrontal cortex. The risk allele was associated with increased expression of HSPG2. The SNP rs 2445142 is located in intron 1 of the HSPG2 gene and is in complete LD with at least six SNPs located from introns 1-9. Among the SNPs associated with TD found in this study, the program TFSEARCH (http:// www.cbrc.jp/research/db/TFSEARCH.html) predicts alteration of the transcription factor, LYF-1, binding affinity between the $\mathrm{T}$ and $\mathrm{C}$ alleles of rs897474 in intron 3 . 
Synonymous SNPs, rs2254357 (exon 6), and rs2254358 (exon 6) that were associated with TD might affect mRNA decay rates. Unfortunately, the mechanism of the association between these SNPs and HSPG2 expression levels could not be elucidated in this study.

From findings in human postmortem brain samples, we speculated that increased expression of HSPG2 is a risk factor for TD and interpreted that decreased expression of Hspg2 in mouse brains after chronic administration of HDL was a compensatory or adaptive response to neuroleptic drugs. We, therefore, hypothesized that decreased expression level of HSPG2 is protective for TD. We examined our hypothesis using hetero-knockout mice and confirmed it after finding lower numbers of VCMs in hetero-knockout mice than in the wild-type littermates after chronic administration of HDL and reserpine. We carried out the experiment using only female mice; therefore, we do not have the data on the sex difference.

The mechanism behind our hypothesis that increased expression levels of HSPG2 may induce a susceptibility to neuroleptic-induced $\mathrm{TD}$ is not known at present. A potential efficacy of cholinergic drugs in the treatment of TD has been reported (Caroff et al, 2001; Tammenmaa et al, 2004). AChE terminates neurotransmission at cholinergic synapses by hydrolyzing acetylcholine. At the neuromuscular junction, AChE is in the basal lamina, where AChE tetramers bind the collagen ColQ, which interacts in turn with the dystroglycan complex through perlecan (Peng et al, 1999). Perlecan is an essential component of the ColQAChE localization in neuromuscular junction (Rotundo et al, 2005). At central synapses, AChE tetramers bind directly to the PRiMA (Perrier et al, 2002). Although ColQ also anchors AChE in brain and heart in addition to skeletal muscle (Feng et al, 1999), the role of perlecan in acetylcholine receptor signaling in central synapses is unclear. In this study, we tested the effect of the AChE inhibitor, physostigmine, on HDL- and reserpon-induced VCMs in mice. We found significant reduction in the number of VCMs only in wild-type mice and the number of VCMs was not reduced in hetero-knockout mice. These findings indicate that perlecan may be involved in the role of AChE in TD and the genotyping and/or levels of HSPG2 may provide useful information about the effectiveness of treatment of TD with AChE.

The other important molecule to which perlecan and TD may be related is FGF2. Perlecan promotes FGF2-FGFR1 binding (Whitelock et al, 1996) and HSPGs including perlecan were upregulated by responding to injury and may have a role in intracellular trafficking of FGF2 in neurons and glia in the adult rat cerebral cortex (Leadbeater et al, 2006). Clozapine increases FGF2 expression and, on the basis of the neuroprotective activity of FGF2, a potential use of clozapine in TD was proposed (Riva et al, 1999).

Perlecan is expressed at the capillary endothelial cells in the brain and perlecan at the blood-brain barrier (BBB) may have a role in maintaining the blood-brain barrier function because of acceptance of the FGF2 secreted from astrocytes (Deguchi et al, 2002). It is reported that neuroleptics, such as HDL and chlorpromazine, alter the blood-brain barrier function and increase brain iron levels, which affect neuroleptic-induced dopamine receptor supersensitivity (Ben-Shachar et al, 1993).
Although the exact mechanisms of the association between HSPG2 and TD are unclear, this study identified the role of HSPG2 in neuroleptic-induced TD.

\section{ACKNOWLEDGEMENTS}

This study was supported by grants from the Mitsubishi Pharma Research Foundation and Kakenhi 20023006 and 20390098. Australian human brain tissues were received from the NSW Tissue Resource Centre, which is supported by The University of Sydney, Neuroscience Institute of Schizophrenia and Allied Disorders, National Institute of Alcohol Abuse and Alcoholism, and the NSW Department of Health.

\section{DISCLOSURE}

The authors declare that no financial support or compensation has been received from any individual or corporate entity over the past 3 years for research or professional service and there are no personal financial holdings that could be perceived as constituting a potential conflict of interest.

\section{REFERENCES}

Araujo NP, Abilio VC, Silva RH, Pereira RC, Carvalho RC, Gonzalez C et al (2004). Effects of topiramate on oral dyskinesia induced by reserpine. Brain Res Bull 64: 331-337.

Arikawa-Hirasawa E, Watanabe H, Takami H, Hassell JR, Yamada Y (1999). Perlecan is essential for cartilage and cephalic development. Nat Genet 23: 354-358.

Association AP (1994). Diagnostic and Statistical Manual of Mental Disorders. 4th Revision. American Psychiatric Association: Washington DC.

Ben-Shachar D, Livne E, Spanier I, Zuk R, Youdim MB (1993). Iron modulates neuroleptic-induced effects related to the dopaminergic system. Isr J Med Sci 29: 587-592.

Burger ME, Fachineto R, Alves A, Callegari L, Rocha JB (2005). Acute reserpine and subchronic haloperidol treatments change synaptosomal brain glutamate uptake and elicit orofacial dyskinesia in rats. Brain Res 1031: 202-210.

Caroff SN, Campbell EC, Havey J, Sullivan KA, Mann SC, Gallop R (2001). Treatment of tardive dyskinesia with donepezil: a pilot study. J Clin Psychiatry 62: 772-775.

de Leon J (2007). The effect of atypical versus typical antipsychotics on tardive dyskinesia: A Naturalistic Study. Eur Arch Psychiatry Clin Neurosci 257: 169-172.

Deguchi Y, Okutsu H, Okura T, Yamada S, Kimura R, Yuge T et al (2002). Internalization of basic fibroblast growth factor at the mouse blood-brain barrier involves perlecan, a heparan sulfate proteoglycan. J Neurochem 83: 381-389.

Devlin B, Roeder K (1999). Genomic control for association studies. Biometrics 55: 997-1004.

Devlin B, Roeder K, Wasserman L (2001). Genomic control, a new approach to genetic-based association studies. Theor Popul Biol 60: $155-166$

Feng G, Krejci E, Molgo J, Cunningham JM, Massoulie J, Sanes JR (1999). Genetic analysis of collagen Q: roles in acetylcholinesterase and butyrylcholinesterase assembly and in synaptic structure and function. J Cell Biol 144: 1349-1360.

Gerlach J (2002). Improving outcome in schizophrenia: the potential importance of EPS and neuroleptic dysphoria. Ann Clin Psychiatry 14: 47-57.

Gerlach J, Casey DE (1988). Tardive dyskinesia. Acta Psychiatr Scand 77: 369-378. 
Haleem DJ (2006). Serotonergic modulation of dopamine neurotransmission: a mechanism for enhancing therapeutics in schizophrenia. J Coll Physicians Surg Pak 16: 556-562.

Inada T, Koga M, Ishiguro H, Horiuchi Y, Syu A, Yoshio T et al (2008). Pathway-based association analysis of genome-wide screening data suggest that genes associated with the gammaaminobutyric acid receptor signaling pathway are involved in neuroleptic-induced, treatment-resistant tardive dyskinesia. Pharmacogenet Genomics 18: 317-323.

Ishiguro $\mathrm{H}$, Koga $\mathrm{M}$, Horiuchi $\mathrm{Y}$, Noguchi $\mathrm{E}$, Morikawa $\mathrm{M}$, Suzuki Y et al (2008). Supportive evidence for reduced expression of GNB1L in schizophrenia. Schizophr Bull. Print copy in press (originally published online 14 November, 2008, at schizophreniabulletin.oxfordjournals.org/cgi/reprint/sbn160v1).

Klawans HL, Goetz CG, Perlik S (1980). Tardive dyskinesia: review and update. Am J Psychiatry 137: 900-908.

Koga M, Ishiguro H, Yazaki S, Horiuchi Y, Arai M, Niizato K et al (2009). Involvement of SMARCA2/BRM in the SWI/SNF chromatin-remodeling complex in schizophrenia. Hum $\mathrm{Mol}$ Genet 18: 2483-2494.

Leadbeater WE, Gonzalez AM, Logaras N, Berry M, Turnbull JE, Logan A (2006). Intracellular trafficking in neurones and glia of fibroblast growth factor-2, fibroblast growth factor receptor 1 and heparan sulphate proteoglycans in the injured adult rat cerebral cortex. J Neurochem 96: 1189-1200.

Malhotra AK, Murphy Jr GM., Kennedy JL (2004). Pharmacogenetics of psychotropic drug response. Am J Psychiatry 161: 780-796.

Menza MA, Grossman N, Van Horn M, Cody R, Forman N (1991). Smoking and movement disorders in psychiatric patients. Biol Psychiatry 30: 109-115.

Muller DJ, Shinkai T, De Luca V, Kennedy JL (2004). Clinical implications of pharmacogenomics for tardive dyskinesia. Pharmacogenomics J 4: 77-87.

Naidu PS, Singh A, Kaur P, Sandhir R, Kulkarni SK (2003). Possible mechanism of action in melatonin attenuation of haloperidolinduced orofacial dyskinesia. Pharmacol Biochem Behav 74: 641-648.
Peng HB, Xie H, Rossi SG, Rotundo RL (1999). Acetylcholinesterase clustering at the neuromuscular junction involves perlecan and dystroglycan. J Cell Biol 145: 911-921.

Perrier AL, Massoulie J, Krejci E (2002). PRiMA: the membrane anchor of acetylcholinesterase in the brain. Neuron 33: 275-285.

Remington G (2007). Tardive dyskinesia: eliminated, forgotten, or overshadowed? Curr Opin Psychiatry 20: 131-137.

Riva MA, Molteni R, Tascedda F, Massironi A, Racagni G (1999). Selective modulation of fibroblast growth factor-2 expression in the rat brain by the atypical antipsychotic clozapine. Neuropharmacology 38: 1075-1082.

Rotundo RL, Rossi SG, Kimbell LM, Ruiz C, Marrero E (2005). Targeting acetylcholinesterase to the neuromuscular synapse. Chem Biol Interact 157-158: 15-21.

Sachdev PS (2000). The current status of tardive dyskinesia. Aust $N$ Z J Psychiatry 34: 355-369.

Saito $\mathrm{T}$, Ishizawa $\mathrm{H}$, Tsuchiya $\mathrm{F}$, Ozawa $\mathrm{H}$, Takahata $\mathrm{N}$ (1986). Neurochemical findings in the cerebrospinal fluid of schizophrenic patients with tardive dyskinesia and neuroleptic-induced parkinsonism. Jpn J Psychiatry Neurol 40: 189-194.

Schooler NR, Kane JM (1982). Research diagnoses for tardive dyskinesia. Arch Gen Psychiatry 39: 486-487.

Tammenmaa IA, McGrath JJ, Sailas E, Soares-Weiser K (2002). Cholinergic medication for neuroleptic-induced tardive dyskinesia. Cochrane Database Syst Rev 3: CD000207.

Tammenmaa IA, Sailas E, McGrath JJ, Soares-Weiser K, Wahlbeck $\mathrm{K}$ (2004). Systematic review of cholinergic drugs for neurolepticinduced tardive dyskinesia: a meta-analysis of randomized controlled trials. Prog Neuropsychopharmacol Biol Psychiatry 28: 1099-1107.

Tarsy D, Baldessarini RJ (2006). Epidemiology of tardive dyskinesia: is risk declining with modern antipsychotics? Mov Disord 21: 589-598.

Whitelock JM, Murdoch AD, Iozzo RV, Underwood PA (1996). The degradation of human endothelial cell-derived perlecan and release of bound basic fibroblast growth factor by stromelysin, collagenase, plasmin, and heparanases. J Biol Chem 271: 10079-10086. 